\title{
Diluente Tris suplementado com óleo de Mauritia flexuoxa sobre a qualidade do sêmen caprino após a criopreservação*
}

\section{Tris diluent supplemented with Mauritia flexuoxa oil on the quality of goat semen after cryopreservation}

\author{
Danilo de Sousa Lima, ${ }^{* *}$ Kenney de Paiva Porfírio, ${ }^{* *}$ Jefferson Hallisson Lustosa da Silva, ${ }^{* *}$ \\ Wallisson Bruno de Morais Pacheco, ${ }^{* *}$ Tuanny Creusa Medeiros Damasceno, ${ }^{* *}$ Letícia Soares de Araújo Teixeira, ${ }^{* *}$ \\ Ana Lys Bezerra Barradas Mineiro, ${ }^{* * *}$ Janaina de Fátima Saraiva Cardoso, ${ }^{* * *}$ Ney Rômulo de Oliveira Paula ${ }^{* * *}$
}

\begin{abstract}
Resumo
Objetivou-se verificar os efeitos, nos parâmetros espermáticos, na integridade mitocondrial, acrossomal e de membrana em células espermáticas, desencadeados pelo uso do Tris (Tris hidroximetil aminometano) suplementado com óleo de Mauritia flexuoxa como diluente para criopreservação de sêmen caprino. Quatro caprinos clinicamente saudáveis foram utilizados. Os animais eram alimentados diariamente com volumoso (Pennisetum purpureum, Schum.), concentrado (ração peletizada com teor de $20 \%$ proteína, $300 \mathrm{~g} /$ animal/dia) e sal mineral específico para Caprinos (Caprinofós ${ }^{\circledR}$ ), à vontade. Dois ensaios foram realizados: I - Teste de toxicidade; II - Criopreservação do sêmen com concentrações ideais. No teste de toxicidade as concentrações avaliadas foram: $5 \%, 10 \%, 15 \%$ e $20 \%$ de diluente a base de óleo de Mauritia flexuoxa. Após o teste de toxicidade, foi escolhido a concentração que apresentou o melhor resultado (5\%). Logo após, foram realizadas mais 32 coletas, que foram diluídas em Tris-gema-glicerol (grupo controle) ou diluente contendo óleo vegetal (Mauritia flexuoxa). As amostras foram criopreservadas com auxílio do aparelho Tk3000 ${ }^{\circledR}$. Após o período mínimo de uma semana as palhetas foram descongeladas em banho-maria a $37^{\circ} \mathrm{C}$ por 30 segundos, acondicionadas em microtubos de centrifugação e homogeneizadas para a análise imediata de motilidade, vigor espermático $\mathrm{e}$ morfologia. Em seguida, por meio de sondas fluorescentes foram avaliadas a integridade de acrossomo, membrana plasmática (Diacetato de Carboxifluresceína e lodeto de Propídeo) e função mitocondrial sob microscopia de epifluorescência. Quanto a motilidade e vigor, integridade mitocondrial e acrossomal, o grupo buriti foi inferior ao grupo controle. O Tris suplementado com óleo de Mauritia flexuoxa na concentração de $5 \%$ não influenciou significativamente a qualidade espermática, porém, observouse morfologia e integridade de membrana favoráveis. Dessa forma, sendo uma alternativa para substituição de diluentes a base de produtos de origem animal.
\end{abstract}

Palavras chave: bode, buriti, espermatozoides.

\begin{abstract}
The objective was to verify the effects, sperm parameters, mitochondrial, acrosomal and membrane integrity in sperm cells, triggered by the use of Tris (Tris hydroxymethyl aminomethane) supplemented with Mauritia flexuoxa oil as a diluent for cryopreservation of goat semen. Four goats clinically healthy were used. The animals were fed daily with bulky (Pennisetum purpureum, Schum.), concentrate (pelleted feed with $20 \%$ protein content, $300 \mathrm{~g} /$ animal / day) and mineral salt Specific for Goats (Caprinofós $囚$ ), ad libitum. Two tests were carried out: I - Toxicity test; II - Semen cryopreservation with ideal concentrations. In the toxicity test as selected were: $5 \%, 10 \%, 15 \%$ and $20 \%$ of Mauritia flexuoxa oil-based diluent. After the toxicity test, the concentration that showed the best result (5\%) was chosen. Soon after, a further 32 samples were obtained, which were diluted in Tris-glycerol (control group) or diluent containing vegetable oil (Mauritia flexuoxa). The samples were cryopreserved using the Tk3000 ${ }^{\circledR}$ machine. After a minimum of one week, the samples were thawed in a $37^{\circ} \mathrm{C}$ water bath for 30 seconds, packed in centrifugation microtubes and homogenized for immediate analysis of motility, sperm vigor and morphology. Then, by means of fluorescent probes, the integrity of the acrosome, plasma membrane (Carboxyflurescein diacetate and Propidium lodide) and mitochondrial function under epifluorescence microscopy were evaluated. As for motility and vigor, mitochondrial and acrosomal integrity, the buriti group was inferior to the control group. Tris supplemented with Mauritia flexuoxa oil at a concentration of $5 \%$ did not significantly influence sperm quality, however, favorable motility, morphology and membrane integrity were observed. Thus, being an alternative to replace diluents based on products of animal origin.
\end{abstract}

Keywords: goat, buriti, sperm.

*Recebido em 19 de novembro de 2019 e aceito em 13 de fevereiro de 2020.

**Universidade Federal do Piauí, Programa de Pós-Graduação em Ciência Animal, Teresina, Piauí, Brasil.

***Universidade Federal do Piauí, Departamento de Clínica e Cirurgia Veterinária, Teresina, Piauí, Brasil.

Autor para correspondência: neyromulo@ufpi.edu.br +55 89999972889. 


\section{Introdução}

A criopreservação de sêmen promove um melhor aproveitamento genético de animais de grandes valores econômicos e zootécnicos (Oliveira et al., 2013). A utilização dessa biotecnologia tem permitido significativos avanços na preservação e multiplicação de várias espécies. No entanto, o processo apresenta distintas limitações, sendo necessárias a evolução e a adaptação de diferentes protocolos de criopreservação de gametas de acordo com as características de cada espécie (Sousa et al., 2014).

A fim de que ocorra a fertilização do ovócito, é extremamente necessário que ocorra a manutenção da membrana plasmática, que pode ser lesada durante o processo de criopreservação, mudando sua fluidez, permitindo a entrada de moléculas que normalmente não entrariam, levando, assim, à morte celular (Oliveira et al., 2013).

O estudo sobre as modificações ocorridas no espermatozoide e em suas membranas, durante a redução da temperatura, elucida possíveis lesões e diminuição da capacidade fertilizante deste gameta masculino. Além disso, possibilita a elaboração de estratégias para reduzir tais injúrias, como a seleção e/ou associação de crioprotetores mais eficazes para determinada espécie, assim como a adição de componentes que podem fornecer substrato ou remover os causadores de lesões celulares, como, por exemplo, as espécies reativas ao oxigênio (Silva e Guerra, 2011).

Nos últimos anos, tem-se observado ampla aplicação de potenciais substâncias adicionadas ao diluente no processo de criopreservação de sêmen em animais domésticos (Sousa et al., 2014). Nesse contexto, diluidores alternativos a base de substratos de origem vegetal, como, por exemplo, a água de coco em pó (ACP), lecitina de soja, óleo de coco e óleo de palma possibilitam um maior controle sanitário do produto, especialmente quando se pretende introduzir o material biológico em diferentes rebanhos dentro do País ou exportá-lo (Bittencourt et al., 2013; Cavalcante et al., 2014; Câmara et al., 2018).

Diante do exposto, o objetivo do presente estudo foi avaliar a eficiência do diluente Tris suplementado com óleo de Mauritia flexuosa sobre a qualidade do sêmen caprino após a criopreservação.

\section{Material e Métodos}

O experimento foi conduzido no Setor de Reprodução Animal do Hospital Veterinário Universitário da Universidade Federal do Piauí, Campus Ministro Petrônio Portela, Cidade de Teresina, Estado do Piauí, Brasil, situado às coordenadas geográficas $5^{\circ}$ $03^{\prime} 23,1^{\prime \prime}$ de Latitude Sul e $42^{\circ} 47^{\prime} 27,9^{\prime \prime}$ de Longitude Oeste, com altitude média de 72,7 metros, e aprovado pela Comissão de ética no uso de animais (CEUA/UFPI) sob o no: 105/15.

Foram utilizados quatro caprinos, com idade média de quatro anos, clinicamente saudáveis e com escore de condição corporal 3,5 , numa escala de (0-5). Os animais foram alimentados diariamente com volumoso (Pennisetum purpureum, Schum.), concentrado (ração peletizada com teor de $20 \%$ proteína, $300 \mathrm{~g} /$ animal/dia) e sal mineral específico para Caprinos (Caprinofós ${ }^{\circledR}$ ) ad libitum. Foram totalizados 64 ejaculados dos quatro bodes utilizados.
A coleta do sêmen foi realizada com o auxílio de vagina artificial específica para pequenos ruminantes, pré-aquecida a uma temperatura média de $39{ }^{\circ} \mathrm{C}$ e acoplada a um tubo coletor graduado (CBRA, 2013). Foram realizadas coletas em duas etapas. Primeiro foi realizado um teste de toxicidade no total de trinta e duas coletas, sendo oito ejaculados de cada animal, os ejaculados foram avaliados com a adição de 5\%,10\%, $15 \%$ e $20 \%$ de diluente a base de óleo de Mauritia flexuoxa extra virgem de origem comercial (Gran Oils, São Paulo), para se verificar qual melhor concentração. As concentrações para o teste de toxicidade foram estabelecidas com base nas concentrações já utilizadas para a gema de ovo. No teste de toxicidade, as amostras de sêmen foram submetidas às diferentes concentrações do óleo e, então, analisadas quanto aos danos de membrana plasmática, motilidade espermática e vigor espermático. A concentração que apresentou o melhor resultado, no teste de toxicidade, foi a de 5\%. Logo após, foram realizadas mais trinta e duas coletas, e os ejaculados foram diluídos em Tris-gema-glicerol (grupo controle) GC ou em diluente contendo óleo vegetal a base de Mauritia flexuoxa (grupo buriti) GB, sendo neste definido a concentração de 5\%. Adicionalmente aos dois grupos citados (GC e GB), foi realizada avaliação antes do processo de criopreservação (avaliação précongelamento), caracterizando o grupo pré-congelamento (GP).

As amostras foram criopreservadas com auxílio do aparelho (TK3000 ${ }^{\circledR}$, TK Tec. Congel. LTDA, Uberaba, MG, Brasil). Posteriormente, as palhetas foram imersas em nitrogênio líquido $\left(-196{ }^{\circ} \mathrm{C}\right)$ e armazenadas em botijões criogênicos. Após o período mínimo de uma semana, as amostras foram descongeladas em banho-maria a $37^{\circ} \mathrm{C}$ por 30 segundos, acondicionadas em microtubos e homogeneizadas para a análise imediata de motilidade, vigor espermático e morfologia, sob microscopia óptica. As avaliações foram realizadas de maneira individual (média por animal) e também de forma geral (média global). Foi avaliada a integridade da membrana espermática com o uso de sondas fluorescentes, conforme descrito por Harrison e Vickers (1990), onde alíquotas de $50 \mu \mathrm{L}$ de sêmen, descongeladas a $37^{\circ} \mathrm{C}$ por 30 segundos e acondicionados em microtubos, foram diluídas em $150 \mu \mathrm{L}$ de TRIS contendo $5 \mu \mathrm{L}$ de Diacetato de Carboxifluoresceína (DCF; Sigma-Aldrich $^{\circledR}$, St. Louis, MO, USA) e $20 \mu \mathrm{L}$ de lodeto de Propídio (IP; Sigma-Aldrich ${ }^{\circledR}$, St. Louis, MO, USA), e incubadas por 10 minutos a $37^{\circ} \mathrm{C}$ para posterior avaliação. A análise deuse pela contagem de 100 espermatozoides em microscópio de epifluorescência (Olympus optical Co., Ltda., Tóquio, Japão) em aumento de 1000 vezes, sob óleo de imersão. As células que apresentaram fluorescência verde foram consideradas íntegras, enquanto aquelas apresentando fluorescência vermelha foram consideradas danificadas.

A atividade mitocondrial foi determinada pela utilização de um fluorocromo catiônico lipofílico JC-1 (Guthrie e Welch, 2006). Para tanto, alíquotas de $50 \mu \mathrm{L}$ de sêmen pós-descongelado foram diluídas em $150 \mu \mathrm{L}$ de Tris contendo $5 \mu \mathrm{L}$ de JC-1 e incubadas por 10 minutos a $37^{\circ} \mathrm{C}$. Um total de 200 espermatozoides foi avaliado em microscópio de epifluorescência (Olympus optical Co., Ltda., Tóquio, Japão), com aumento de 1000x, sob óleo de imersão. As células coradas em laranja foram classificadas com alto potencial de membrana mitocondrial, enquanto aquelas coradas em verde foram classificadas com baixo potencial de membrana. 
Para análise da integridade acrossomal foi utilizado o isocianato de fluoresceína conjugado (FITC-PNA; Sigma-Aldrich ${ }^{\circledR}$, St Louis, MO, USA), de acordo com a técnica descrita por Roth et al. (1998), em que uma alíquota de $20 \mu \mathrm{L}$ da solução estoque de FITC-PNA foi descongelada e adicionada a $480 \mu \mathrm{L}$ de solução de fosfato tamponada (PBS). Alíquotas de $20 \mu \mathrm{L}$ dessa solução foram colocadas sobre esfregaços de lâminas contendo espermatozoides, as quais foram incubadas por 20 minutos em câmara úmida a $4{ }^{\circ} \mathrm{C}$, na ausência de luz. Após incubação, as lâminas foram enxaguadas em PBS refrigerado $\left(4^{\circ} \mathrm{C}\right) \mathrm{e}$ colocadas para secagem na ausência de luz. Imediatamente antes da avaliação, $5 \mu \mathrm{L}$ de meio de montagem UCD foi colocado sobre lâmina e coberta com lamínula. Foram avaliados 200 espermatozoides, em aumento de 1000x sob óleo de imersão, usando microscopia de epifluorescência (Olympus optical Co., Ltda., Tóquio, Japão). As células espermáticas foram classificadas como portadoras de acrossomas intactos (AI), quando a região acrossomal apresentou fluorescência verde intensa. Foram considerados acrossomas lesionados quando as células espermáticas não apresentaram coloração verde ou a fluorescência verde se restringiu apenas à região equatorial da cabeça espermática.

A análise de variância (ANOVA) foi realizada por meio do software SAS 9.0, e para comparação de médias foi utilizado o teste de Tukey a $5 \%$.

\section{Resultados e Discussão}

De acordo com o teste de toxicidade (do óleo vegetal de Mauritia flexuosa), obteve-se melhor resultado na concentração de 5\%, ou seja, essa concentração foi a menos tóxica para as células espermáticas. Ao passo que, as demais concentrações $(10 \%$, $15 \%$ e $20 \%$ ) foram tóxicas para as células espermáticas, e desta forma não foram utilizadas neste estudo.

As características microscópicas do sêmen pós-descongelamento acrescidos ou não de Óleo de Mauritia flexuosa estão descritos na tabela 1.

$\mathrm{Na}$ tabela 1 verifica-se que os parâmetros motilidade e vigor do grupo controle (GC) foram superiores aos observados no grupo buriti (GB5\%), apresentando diferença significativa no teste de Tukey a 5\% de probabilidade. Em relação ao parâmetro morfologia ambos os grupos não apresentaram diferença significativa.

Os resultados referentes as análises através de sondas fluorescentes estão descritas na tabela 2 .

Neste estudo, não houve diferença entre os grupos testados quanto a avaliação da integridade da membrana plasmática através do uso de sondas florescentes (Tabela 2) (Figura 1). Isso é bastante satisfatório, uma vez que, para uma boa taxa de fertilidade, a membrana espermática deve estar íntegra para que a fecundação seja possível. Quanto à integridade mitocondrial e acrossomal, o grupo controle foi superior ao grupo buriti (Tabela 2).

Observou-se que os parâmetros motilidade e vigor do grupo buriti (animal 1), foram inferiores aos encontrados no grupo controle.
Tabela 1: Características seminais (Médias \pm Desvio padrão) no pré-congelamento e pós-descongelamento do sêmen de bodes acrescidos ou não de Óleo de Mauritia flexuosa (5\%)

\begin{tabular}{lccc}
\hline Parâmetros & GP & GC & GB5\% \\
\hline Motilidade & $63,75 \pm 19,18^{\mathrm{a}}$ & $36,25 \pm 8,94^{\mathrm{b}}$ & $18,15 \pm 13,63^{\mathrm{c}}$ \\
Vigor & $2,53 \pm 0,51^{\mathrm{a}}$ & $2,34 \pm 0,48^{\mathrm{a}}$ & $1,74 \pm 1,24^{\mathrm{b}}$ \\
Morfologia & $96,09 \pm 1,33^{\mathrm{a}}$ & $96,03 \pm 1,35^{\mathrm{a}}$ & $96,03 \pm 1,33^{\mathrm{a}}$ \\
& & & \\
\hline
\end{tabular}

*Médias seguidas de mesma letra nas linhas não diferem entre si pelo Teste de Tukey a 5\% de probabilidade. GP: Grupo pré-congelamento; GC: Grupo Controle; GB5\%: Grupo Buriti, concentração 5\%.

Tabela 2: Características seminais (Médias \pm Desvio padrão) para integridade de membrana plasmática, integridade mitocondrial e integridade do acrossoma, pós-descongelamento de espermatozoides caprinos acrescidos ou não de Óleo de Mauritia flexuosa

\begin{tabular}{lcc}
\hline \multicolumn{1}{c}{ Parâmetros } & GC & GB5\% \\
\hline Membrana plasmática (\%) & $49,72 \pm 21,96^{\mathrm{a}}$ & $37,84 \pm 26,67^{\mathrm{a}}$ \\
Mitocôndrias (\%) & $34,28 \pm 27,50^{\mathrm{a}}$ & $13,31 \pm 20,94^{\mathrm{b}}$ \\
Acrossoma (\%) & $39,25 \pm 19,29^{\mathrm{a}}$ & $26,15 \pm 18,76^{\mathrm{b}}$ \\
\hline
\end{tabular}

*Médias seguidas de mesma letra nas linhas não diferem entre si pelo Teste de Tukey a 5\% de probabilidade. GC: Grupo Controle; GB5\%: Grupo Buriti, concentração $5 \%$.

Em contrapartida, nos animais 2, 3 e 4, apenas o parâmetro motilidade do grupo buriti foi inferior ao grupo controle (Tabela 3). Semelhante ao que foi descrito no trabalho de Del Valle et al. (2013), onde adicionaram ao diluidor, óleo de coco e óleo de palma, para seguir ao processo de criopreservação do sêmen e verificaram que os resultados foram inferiores aos encontrados no sêmen congelado com $20 \%$ de gema de ovo no diluidor.

O grupo buriti (animais 1, 2, 3 e 4) apresentou membrana plasmática íntegra após o processo de congelamento. A integridade mitocondrial do grupo buriti (animais 1, 2 e 3) foi inferior ao grupo controle. A integridade acrossomal do grupo buriti (animais 2 e 3) foi inferior ao grupo controle (Tabela 3).

Os parâmetros observados no grupo controle estão de acordo com as características mínimas preconizadas pelo Colégio Brasileiro de Reprodução Animal para o sêmen caprino congelado (CBRA, 2013). Para se obter esses parâmetros fisiológicos adequados e bons resultados após a criopreservação, Silva e Guerra (2011) relatam que faz se necessária a padronização de protocolos de criopreservação para cada espécie animal.

Autores como Oliveira et al. (2013) e Medina et al. (2000) relatam que alguns animais possuem algumas particularidades em seus ejaculados o que pode torná-los mais resistentes quando comparados a outros animais. Em vista disso no presente trabalho também foi avaliado cada animal individualmente, tabela 3 , para se determinar se existia alguma variação entre animais. 

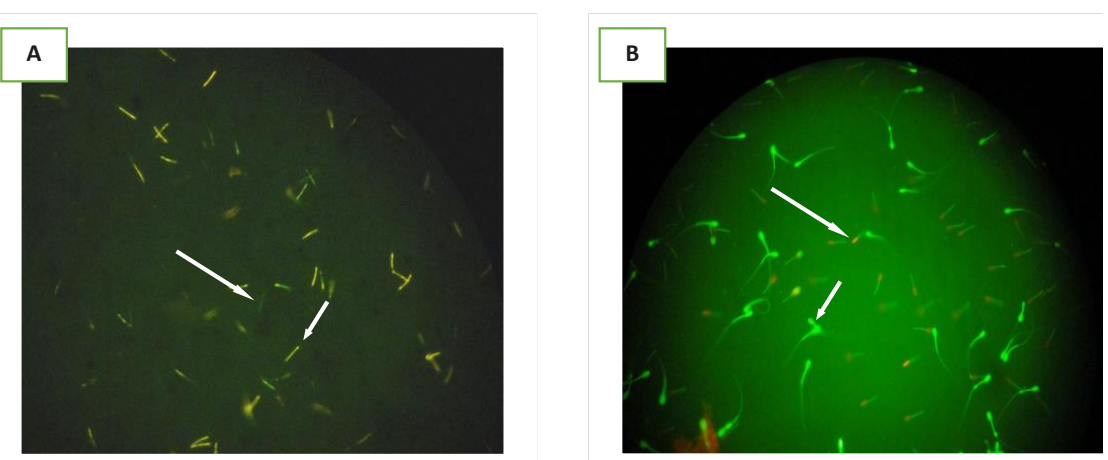

Figura 1: Avaliação da Integridade mitocondrial em A: Alto potencial (seta curta) e baixo potencial (seta longa) da membrana mitocondrial com uso de sonda fluorescente JC-1. Integridade de membrana em B: Células portadoras de acrossomas intactos (seta curta) e lesionados (seta longa), através do uso das sondas florescentes DCF + IP. Aumento 1000x.

Tabela 3: Avaliação dos parâmetros espermáticos por animal (Médias \pm Desvio padrão) e avaliação da integridade dos espermatozoides através do uso de sondas fluorescentes

\begin{tabular}{|c|c|c|c|c|c|c|c|c|}
\hline \multirow{3}{*}{ Parâmetros } & \multicolumn{8}{|c|}{ Animais } \\
\hline & \multicolumn{2}{|c|}{1} & \multicolumn{2}{|c|}{2} & \multicolumn{2}{|c|}{3} & \multicolumn{2}{|c|}{4} \\
\hline & GC & GB5\% & GC & GB5\% & GC & GB5\% & GC & GB5\% \\
\hline Motilidade & $40,01 \pm 7,5^{a}$ & $19,37 \pm 15,69^{b}$ & $32,5 \pm 7,9^{a}$ & $18,1 \pm 11,70^{b}$ & $37,01 \pm 9,35^{a}$ & $15,12 \pm 13,55^{b}$ & $35,01 \pm 9,01^{a}$ & $20 \pm 12,74^{b}$ \\
\hline Vigor & $2,64 \pm 0,48^{a}$ & $1,62 \pm 1,11^{b}$ & $2,25 \pm 0,43^{\mathrm{a}}$ & $1,75 \pm 1,08^{a}$ & $2,25 \pm 0,4^{a}$ & $1,75 \pm 1,08^{a}$ & $2,37 \pm 0,44^{a}$ & $1,87 \pm 1,16^{a}$ \\
\hline Morfologia & $95,62 \pm 1,21^{a}$ & $95,55 \pm 1,00^{\mathrm{a}}$ & $96,37 \pm 1,31^{\mathrm{a}}$ & $96,25 \pm 1,19^{a}$ & $95,87 \pm 1,45^{a}$ & $95,62 \pm 1,21^{a}$ & $96,25 \pm 1,29^{a}$ & $96,75 \pm 1,47^{\mathrm{a}}$ \\
\hline Membrana plasmática (\%) & $45,5 \pm 16,47^{\text {a }}$ & $48,2 \pm 30,82^{a}$ & $51,75 \pm 25,2^{\mathrm{a}}$ & $43,8 \pm 26,40^{a}$ & $50 \pm 22,7^{a}$ & $31,6 \pm 19,51^{\text {a }}$ & $51,63 \pm 25,3^{\text {a }}$ & $27,63 \pm 25,6^{a}$ \\
\hline Mitocôndrias & $22,50 \pm 20,8^{a}$ & $4,25 \pm 3,67^{b}$ & $31,8 \pm 22,3^{a}$ & $8,87 \pm 13,0^{b}$ & $64,2 \pm 30,3^{a}$ & $7,25 \pm 9,79^{b}$ & $35,375 \pm 29,1^{a}$ & $32,8 \pm 30,5^{\mathrm{a}}$ \\
\hline Acrossoma (\%) & $29,12 \pm 21,37^{a}$ & $36,6 \pm 21,64^{\text {a }}$ & $40,5 \pm 18,4^{a}$ & $19,0 \pm 10,9^{b}$ & $50,8 \pm 10,0^{a}$ & $25,1 \pm 18,0^{b}$ & $36,5 \pm 21,5^{\mathrm{a}}$ & $23,8 \pm 21,3^{a}$ \\
\hline
\end{tabular}

*Médias seguidas de mesma letra nas linhas não diferem entre si pelo Teste de Tukey a 5\% de probabilidade. GC: Grupo Controle; GB5\%: Grupo Buriti, concentração $5 \%$.

*Integridade dos espermatozoides (Normais).

A baixa fertilidade do sêmen descongelado é atribuída, em grande parte, as alterações por que passam as membranas espermáticas (Plasmáticas, acrossomais e mitocondriais) durante os processos de congelação e descongelação (Medina et al., 2000). Neste contexto as análises através de sondas fluorescentes tornam-se essenciais para verificar se há ou não alterações que possam tornar os espermatozoides inaptos a fertilização.

De acordo com Snoeck et al. (2007), os espermatozoides devem possuir membrana e organelas íntegras e funcionais e um genoma haploide intacto para obtenção de sucesso na concepção.

Medina et al. (2000), utilizando sondas fluorescentes para a avaliação da integridade da membrana de espermatozoides ovinos verificaram que o número de membranas integras aumentaram na medida que se aumentava os níveis de testosterona.

Em relação avaliação da viabilidade do acrossoma os resultados desse trabalho são semelhantes ao encontrados por Medina et al. (2000), onde os autores afirmam que o principal motivo de acrossoma danificado seria a influência dos danos causados pela criopreservação.

De acordo com Oliveira et al. (2013), cada animal possui uma pequena variação na composição da membrana plasmática o que pode torná-lo mais resistente ou não ao processo de criopreservação. Porém, no presente trabalho não houve diferença entre os animais na avaliação da membrana plasmática, mitocôndria e acrossoma.

Em relação à análise morfológica do acrossoma, Medina et al. (2000) observaram diferenças entre animais e entre tratamentos e também destacaram a influência da técnica de criopreservação sobre a incidência de acrossoma danificados.

O óleo de buriti (Mauritia flexuosa) pode ser utilizado em tecnologias aplicadas ao sêmen animal, pois é rico em esteróis, fosfatídeos, vitaminas lipossolúveis (lal, 2005), ácidos graxos saturados e polisaturados (Costa et al., 2011), além dos macro e micro minerais como cálcio, potássio, zinco e fósforo (Darnet, et al., 2011), no entanto, são necessários mais estudos para otimizar seu uso. Vale ressaltar que um dos requisitos para o sucesso de sua aplicação como diluidor e crioprotetor é manter a viabilidade da célula espermática e prevenir as crioinjúrias durante a congelação e descongelação seminal (Salmito Vanderley et al., 2012).

Neste trabalho, os resultados mostraram que a adição do óleo de buriti ao diluente e crioprotetor seminal, apesar dos baixos resultados, fomentam a realização de mais estudos a fim de efetivar a substituição da gema de ovo como crioprotetor. O uso de diluente de origem vegetal influencia positivamente na questão sanitária, uma vez que o grande gargalo da utilização da gema de ovo é a ausência de controle sanitário por se tratar de um produto de origem animal. 
Gil et al. (2003) relatam que apesar da gema de ovo beneficiar os espermatozoides na criopreservação, por ser um produto de origem animal pode apresentar risco ao potencial das células, por conter agentes microbiológicos específicos, comprometendo assim a qualidade espermática. Relatam também a substituição da gema de ovo por produtos de origem vegetal como a lecitina de soja onde vários autores como Khalifa et al. (2013), obtiveram bons índices de fertilidade (77\%) e observaram a sua interação com a idade do animal, bem como Fukui et al. (2008) que obtiveram resultados semelhantes de índices de fertilidade, na substituição do produto de origem animal (58\%) pelo de origem vegetal $(56 \%)$, além de destacar a importância da lecitina de soja para minimizar os riscos sanitários.

Corroborando com os resultados deste estudo, Cavalcante et al. (2014) utilizando um produto vegetal a base de Água de Coco em Pó (ACP-102 ${ }^{\circledR}$ ), verificaram que o uso desse produto conferiu uma menor proteção aos espermatozoides de ovinos quando comparado ao grupo controle contendo o Tris. Porém, os autores também afirmam que seu uso pode se tornar uma alternativa viável desde que haja um aprimoramento de sua fórmula.

\section{Referências}

BITTENCOURT, R.F; OBA E; FILHO A.L.R; CHALHOUB M; AZEVEDO H.C; BICUDO S.D. Avanços na criopreservação do sêmen ovino I: Diluidores e crioprotetores. Ciência Animal Brasileira. v.14, n.4, p.522-536, 2013.

CÂMARA T.S.; NUNES T.G.P.; TONIOLLI R. Diluentes seminais para pequenos ruminantes. Ciência Animal, v.28, n.2, p.67-83, 2018.

CAVALCANTE, J.M.; BRASIL, O.O.; SALGUEIRO, C.C.M.; SALMITO-VANDERLEY.; NUNES,J.F. Criopreservação do sêmen ovino em meio diluente à base de água de coco em pó (ACP 102). Ciência Animal Brasileira, v.15, p.344-353, 2014.

COLÉGIO, B.R.A. Manual para Exame Andrológico e Avaliação de Sêmen Animal. $3^{\mathrm{a}}$ edição. Belo Horizonte, 2013.

COSTA, P. A.; BALLUS, C. A.; TEIXEIRA FILHO, J.; GODOY, H. T. Fatty acids profile of pulp and nuts of Brazilian fruits. Ciência e Tecnologia de Alimentos, v.31, n.4, p.950-954, 2011.

DARNET, S. H.; SILVA, L. H. M.; RODRIGUES, A. M. C.; LINS, R. T. Nutritional composition, fatty acid and tocopherol contents of buriti (Mauritia flexuosa) and patawa (Oenocarpus bataua) fruit pulp from the Amazon region. Food Science and Technology, v.31, p.488-491, 2011.

DEL VALLE, I.; SOUTER, A.; MAXWELL, W.M.C.; MUIÑOBLANCO, T.; CEBRIÁN-PEREZ, J.A. Function of ram spermatozoa frozen in diluentes supplemented with casein and vegetable oils. Animal Reproduction Science, v.138, n3-4, p.213-219, 2013.

FUKUI, Y.; KOHNO, H.; TOGARI, T.; HIWASA, M.; OKABE, K. Fertility after artificial insemination using a soybean-based semen extender in sheep. Journal of Reproduction and Development, v.54, n.4, p.286-289, 2008.

GIL, J; RODRIGUEZ-IRAZOQUI, M; LUNDEHEIM, N; SÖDERQUIST, L; RODRÍGUEZ-MARTÍNEZ, H. Fertility of ram semen frozen in Bioexcell and used for cervical artificial insemination. Theriogenology. v.59, p.1157-1170, 2003.

GUTHRIE, H.D; WELCH, G.R. Determination of intracellular reactive oxygen species and high mitochondrial membrane potential in Percoll-treated viable boar sperm using fluorescenceactivated flow cytometry. Journal of Animal Science. v.84, p.20892100, 2006.
Alguns autores como Oliveira et al. (2013) e Medina et al. (2000) relatam que alguns animais possuem determinadas particularidades em seus ejaculados que podem torná-los mais resistentes quando comparados a outros animais. Em vista disso, no presente trabalho, foram avaliados cada animal individualmente para determinar a existência de variação entre animais.

\section{Conclusão}

O uso do crioprotetor Tris (Tris hidroximetil aminometano) suplementado com óleo de Mauritia flexuoxa na concentração de $5 \%$ apresentou menor toxidade em relação às demais concentrações avaliadas, no entanto, não contribuiu positivamente na qualidade do sêmen após descongelação quando comparado ao grupo controle (com gema de ovo). Nesse sentido, novos estudos são necessários, uma vez que a substituição de diluentes de origem animal por diluentes de origem vegetal constitui-se de uma boa alternativa.

HARRISON, R.A.P; VICKERS, S.E. Use of fluorescent probes to assess membrane integrity in mammalian spermatozoa. Journal Reproduction and Fertility. v.88, p.343-352, 1990.

IAL. INSTITUTO ADOLFO LUTZ. Normas Analíticas do Instituto Adolf Lutz. Métodos químicos e físicos para análise de alimentos, $4^{\mathrm{a}}$ ed., São Paulo: Instituto Adolfo Lutz, 2005.

KHALIFA, T.; LYMBEROPOULOS, A.; THEODOSIADOU, E. Association of soybean-based extender with field fertility of stored ram (Ovis aries) semen: A randomized double-blind parallel group design. Theriogenology, v.79, n.3, p.517-527, 2013.

MEDINA, V.H; VICENTE, W.R.R; ESPER, C.R; MALHEIROS, E.B. Uso de sondas Fluorescentes para avaliação da integridade da membrana plasmática de espermatozoides ovinos antes e após a criopreservação. ARS Veterinária. v.16, p.204-209, 2000.

OLIVEIRA, C.G; OLIVEIRA, B.M.M; CELEGUINE, E.C.C; FERNANDES, C.B; MATOS, C.B. Criopreservação do sêmen equino: uma revisão. Revista Brasileira de Reprodução Animal. v.37, p.23-28, 2013.

ROTH, T.L; WEISS, R.B; BUFF, J.L. Heterologous in vitro fertilization and sperm capacitation in an endangered African antelope, the Scimitar-Horned Oryx (Oryx dammah). Biology of Reproduction v.58, p.475-482, 1998.

SALMITO-VANDERLEY, C.S.B; VIEIRA, M.J.A.F; LEITE, L.V; OLIVEIRA, F.C.E; LINHARES, F.R.A; SALGUEIRO, C.C.M; NUNES, J.F. Meios de congelação para conservação de sêmen de peixes da família Characidae. Ciência Animal. v.22, p.255-268, 2012.

SILVA, S.V; GUERRA, M.M.P. Efeitos da criopreservação sobre as células espermáticas e alternativas para redução das crioinjúrias. Revista Brasileira de Reprodução Animal. v.35, p.370384, 2011.

SOUSA, A.L.P; LIMA, G.L; SILVA, A.R. Alternativas para o aperfeiçoamento dos protocolos de criopreservação de sêmen de animais selvagens. Revista Brasileira de Reprodução Animal. v.38, p.98-102. 2014.

SNOECK, P.P.N; HENRY, M; MELO, M.I.V. Efeito de diferentes diluidores sobre a viabilidade espermática pós descongelamento de sêmen equino. Arquivo Brasileiro de Medicina Veterinária e Zootecnia. v.59, p.56-64, 2007. 